\title{
Enteroparasitosis infections among renal transplant recipients in Khartoum state, Sudan 2012-2013
}

\author{
Nouh Saad Mohamed 1,2,3* Emmanuel Edwar Siddig 4,5,6, Mona Ali Mohamed³, \\ Basma AbdlMoniem Alzein ${ }^{6}$, Hanaa Hashim Saeed Osman7 , Emmanuel E. Tanyous ${ }^{8}$, Bahaeldin K. Elamin 9,10 \\ and Ali Mahmoud Mohammed Edris ${ }^{4,11}$
}

\begin{abstract}
Objectives: Renal transplantation procedure markedly increased over the past few decades. The risk of harboring parasitic diseases may affect transplant recipients during life expectancy. We aimed in this study to determine the enteroparasitosis frequency among renal transplant recipients in Khartoum state, Sudan. A case-control hospitalbased study performed between November 2012 and May 2013, on 300 renal transplant recipients attending Sudanese Kidney Association hospital in Khartoum state, Sudan, along with 300 normal healthy individuals matching the case in age and sex. Stool samples were collected for parasitological studies.

Results: Out of the 300 renal transplant recipients: 242 (80.7\%) were males mean age $43 \pm 11.28$ and 58 (19.3\%) were females mean age $41 \pm 13.41$. Intestinal parasitic infection was observed in 118 participants and the overall frequency was 19.7\%; of which 64 were cases (21.3\%) and 54 (18.0\%) were controls. Eight different species of intestinal parasites were identified; Entamoeba histolytica/dispar (7.5\%), Entamoeba coli (6.5\%), Giardia lambelia (3.2\%), Cryptosporidium parvum (1.2\%), Ascaris lumbricoides (0.6\%), Enterobius vermicularis (0.3\%), (0.2\%) for each of Strongyloides stercoralis and Hymenolepis nana.
\end{abstract}

Keywords: Renal transplant, Parasitic infections, Khartoum, Sudan

\section{Introduction}

Renal transplantation has significantly increased over the past few decades [1]. In 2013, 75,000 kidney transplants were done worldwide in the year 2010 [2]. It is also the only treatment means for devastating stage of renal failure [3]. However, the long-lasting endurance of renal tissues so far remains a baffling and unsolved question as the majority of the renal transplant recipients go through failure within a decade of their transplantation [4].

Parasitic infections occurring in renal transplant recipients are still of neither unknown prevalence nor incidence hence the number of infected patients showing symptoms are few [5]. Remarkably, several classes of

\footnotetext{
*Correspondence: Nouh_Saad@outlook.com

${ }^{1}$ Department of Parasitology and Medical Entomology, Faculty of Medical

Laboratory Sciences, Sinnar University, Sinnar, Sudan

Full list of author information is available at the end of the article
}

parasites occur in allograft recipients, however the number of pathogenic parasites that could infect transplant recipients is about $5 \%$ and still does not reflect the actual incidence of the parasitic infections, however it is only for those causing transplanted organ to be rejected [6-8].

Importantly, parasitic infections could be considered as a major cause of distressing and death after transplantation [9]. Surprisingly, the incidence of first infections in the earliest 3 years ensuing kidney transplantation is 45.0 per 100 patient-years of follow-up [10]. Oddly, emerging intestinal parasites have become a significant opportunistic pathogens accountable for the most clinically important infections in immune-compromised patients especially renal transplant patients [11, 12]. Although parasites might be occupied through the fecooral route, it is also might be reactivated in the immunocompromised host infected transplanted organ or being 
as dormant stages in the recipient itself [6], or by means of acquired transmission occurred by transplanted organ into a naive recipient [1]. Intestinal parasitic infections among renal transplant recipients requires careful deliberation as the infection may exacerbate with difficulties upon treatment [5]. Immunosuppressive drugs used by renal transplant recipient were reported to increase the susceptibility of harboring parasitic infection [11]. Several studies discussed presence of certain parasites detected in immunocompromised patients specially transplant recipients [11, 13-15].

Remarkably, opportunistic parasitic infections occurring in renal allograft recipients are well-known agents not only for causing diarrhea, but also are most important public health problem in developing countries [16]. Furthermore, intestinal parasitosis has been documented as a clinically significant infection not merely in immunocompromised patients, but also in hemodialysis [17-19].

In Sudan, intestinal parasites were transmitted via contaminated fresh vegetables that eaten on daily basis [20], indicating that public health being at high risk of infection with intestinal parasites, resulting in the increase of parasitic diseases harboring by susceptible immunocompromised patients particularly those of renal transplantation. Nevertheless, to our knowledge there have been very scarce studies addressing this issue in Sudan [21]. The aim of this study is to determine the frequency of enteroparasitosis among renal transplant recipients compared to healthy individuals in Khartoum state, Sudan.

\section{Main text}

\section{Materials and methods}

A case-control hospital-based study carried out in a period between November 2012 and May 2013; 300 renal transplant recipients were enrolled in the study while they attending Sudanese Kidney Association hospital in Khartoum state, Sudan. Another 300 normal individuals were recruited from the general population; taking into consideration the absence of any intestinal symptoms. Matching of cases and controls in terms of socio-economic and living condition status has been done through questionnaire survey for the recruited controls, and to ensure that the subjects chosen to participate as healthy have not undergone renal transplantation in the past. Stool specimens were collected in sterile, screw capped disposable plastic containers, and immediately transported to the Department of Microbiology-University of Khartoum, for parasitological studies. Additionally, dialysis duration and previous usage of immunosuppressive medications or any other medications gleaned were recorded using a well-designed questionnaire. Direct smears were applied to the patients' and the healthy participants' samples on a clean slide using a wooden stick, then immediately two drops of normal saline were added, and gently mixed with the specimen. Then the specimens were covered by coverslip and examined using a low power objective $(\times 10)$ and $(\times 40)$ for identification of parasites. Moreover, formalinethyl acetate concentration technique was performed. In this context the sediments were examined for intestinal protozoa, eggs and larvae of intestinal helminths using light microscope [22]. Another smear was prepared and stained by modified Ziehl-Neelsen acid-fast stain for the detection of Cryptosporidium oocyst according to guidelines by Casemore et al. [23].

\section{Statistical analysis}

Data analysis was performed using the Statistical Package for Social Sciences (SPSS) v20.0. Chi square test was used to find out the association between parasitic infections among renal transplant and healthy group. Additionally, odds ratio for both case and control groups for harboring intestinal parasites were also been calculated.

\section{Results}

\section{Study characteristics}

A total of 600 fecal samples were collected during this study. 300 were renal transplant recipients their ages ranged between 11 and 80 years with a mean age of $43 \pm 13.4$ years. Of these, $242(80.7 \%)$ were males mean age $43 \pm 11.28$ and $58(19.3 \%)$ were females mean age $41 \pm 13.41$, while the healthy participants were 300 individuals, their age ranges between 9 and 75 years with a mean age of $44 \pm 13.1$ years. Of these 255 (85\%) were males mean age $50 \pm 19.18$, 48 (16\%) were females mean age $43 \pm 13.84$. Most of the study participants were categorized as middle income (68.6\%) 206 were cases and 206 controls, while (31.4\%) were of low income condition; 100 were cases and 88 were controls. No significant found for the association of socio-economic status with the prevalence of intestinal parasitosis, P value 0.503.

Family history of renal failure was reported among 39 (13.0\%) of renal transplant recipients, while none of the participated controls had any family history of renal failure, $\mathrm{P}$ value 0.000 . Also, all transplanted recipients assigned for dialysis before transplantation process, their dialysis periods were vary; 193 (64.4\%) assigned for less than 3 years, 74 (24.6\%) from 4 to 7 years, 22 (7.3\%) from 8 to 12 years, 8 (2.7\%) from 13 to 16 years and for more than 16 years there was only one recipient.

All renal transplant recipients were using several combinations of treatment; Cyclosporine with Cortisone were 62 (20.7\%), Cyclosporine with Azathioprine were 116 (38.6\%), Tacrolimus combined with Mycophenolate mofetil were 83 (27.7\%) and 39 (13.0\%) were using Cyclosporine combined with Cortisone and Mycophenolate mofetil. 
Anti-parasitic medications used by the renal transplant recipients and the healthy controls were vary depending on the causative agent of the enteroparasitosis; mostly intestinal protozoa was treated using Metronidazole tabs taking into consideration the increase in blood level of cyclosporine, therefore kidney function monitoring was done while taking the course of medication. While all infections caused by helminthes parasites were treated with Praziquantel, and infections caused by the roundworms were treated with Mebendazole. Nevertheless, cryptosporidium infections were treated by Nitazoxanide.

\section{Enteroparasitosis prevalence}

A total of 118/600 (19.7\%) stool samples were found to be positive for intestinal parasitic infection, 64/300 (21.3\%) belong to renal transplant recipients and 54/300 (18.0\%) to the considered healthy participants. Odds ratio for harboring intestinal parasites among the case and controls was 1.24 (CI 95\%, 0.83-1.86). Interestingly, of both renal transplant and healthy participants, the most detected parasites were Entamoeba histolytica/dispar with frequency of 24 (37.5\%) and 21 (38.9\%) respectively. No significant difference in presence of E. histolytica/dispar infections between cases and controls $(\mathrm{P}>0.05)$. The frequency of other detected intestinal parasites in renal transplant recipients include Entamoeba coli 21 (32.8\%), Giardia lambelia 9 (14.1\%), Cryptosporidium parvum 5 (7.81\%), Ascaris lumbricoides 2 (3.12\%), Enterobius vermicularis 1 (1.5\%), Strongyloides stercoralis 1 (1.5\%), and Hymenolepis nana 1 (1.5\%). Concerning control group, the most detected parasites among the 54 positive stool samples were E. coli 18 (33.3\%), G. lambelia were (18.51\%), C. parvum (3.7\%), A. lumbricoides (3.7\%), and E. vermicularis $(1.85 \%)$. Across the total studied participants there were no significant differences between transplanted recipients and control groups in the frequency distribution of intestinal parasites infecting both groups (Table 1).

Concerning the infected renal transplant recipient, intestinal parasitosis was more prevalent among recipient taking Cyclosporine with Azathioprine treatments $28(43.8 \%)$, while recipients taking the combination of Cyclosporine combined with Cortisone and Mycophenolate mofetil were the least group showing intestinal infection; 7 (10.9\%) (Fig. 1).

The illustration of intestinal parasites infecting the renal transplant recipient, E. histolytica was found to be the most prevalent parasite followed by E. coli; 24 (37.5\%), 21 (32.8\%) respectively. The frequencies of the other parasites were shown in Table 2.

\section{Discussion}

Frequency of intestinal parasites in renal transplant recipients is not well-known in Sudan, so we had to compare our results to other studies carried out on renal transplant recipients worldwide. In the present study, E. histolytica/dispare was the most prevalent parasite detected in both groups, followed by E. coli and

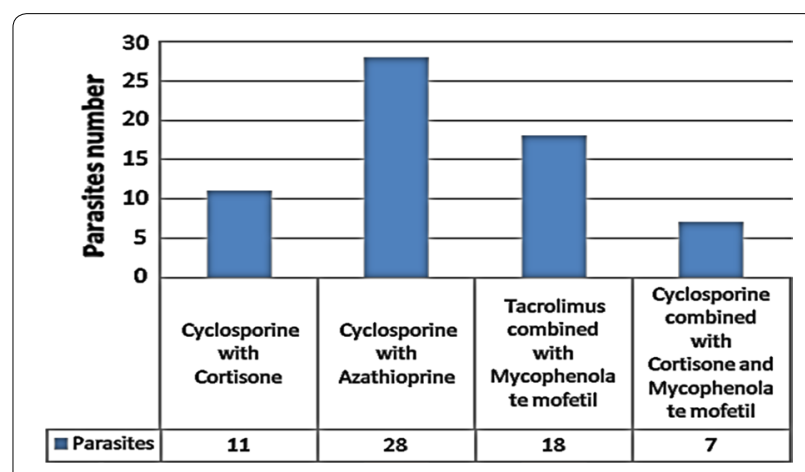

Fig. 1 The frequency of intestinal parasitosis among the different regimens groups used for the treatment of the renal transplanted recipients

Table 1 Illustrate the intestinal parasites distribution across the study population

\begin{tabular}{|c|c|c|c|c|}
\hline Parasites detected & Transplant recipients & Healthy participants & Total & $P$ value \\
\hline Negative & $236(78.7 \%)$ & $246(82.0 \%)$ & $482(80.3 \%)$ & NA \\
\hline Entamoeba histolytica/dispar & $24(8.0 \%)$ & $21(7.0 \%)$ & $45(7.5 \%)$ & 0.756 \\
\hline Entamoeba coli & $21(7.0 \%)$ & $18(6.0 \%)$ & $39(6.5 \%)$ & 0.740 \\
\hline Cryptosporidium parvum & $5(1.7 \%)$ & $2(0.7 \%)$ & $7(1.2 \%)$ & 0.450 \\
\hline Giardia lambelia & $9(3.0 \%)$ & $10(3.3 \%)$ & $19(3.2 \%)$ & 1.000 \\
\hline Enterobius vermicularis & $1(0.3 \%)$ & $1(0.3 \%)$ & $2(0.3 \%)$ & 1.000 \\
\hline Hymenolepis nana & $1(0.3 \%)$ & 0 & $1(0.2 \%)$ & 1.000 \\
\hline Ascaris lumbricoides & $2(0.7 \%)$ & $2(0.7 \%)$ & $4(0.7 \%)$ & 1.000 \\
\hline Strongyloides stercoralis & $1(0.3 \%)$ & 0 & $1(0.2 \%)$ & 1.000 \\
\hline Total & $300(50.0 \%)$ & $300(50.0 \%)$ & $600(100 \%)$ & \\
\hline
\end{tabular}


Table 2 Frequency of intestinal parasites among the different regimens used with renal transplant recipients

\begin{tabular}{|c|c|c|c|c|c|c|c|c|c|}
\hline Treatment & E. $H / D$ & E. C & C.P & G. L & E. V & H. N & A. $L$ & S. S & Total \\
\hline Cyclosporine with Cortisone & $3(27.3 \%)$ & $5(45.5 \%)$ & $0(0.0 \%)$ & $2(18.2 \%)$ & $0(0.0 \%)$ & $0(0.0 \%)$ & $1(9.1 \%)$ & $0(0.0 \%)$ & $11(17.2 \%)$ \\
\hline Cyclosporine with Azathioprine & $11(39.3 \%)$ & $5(17.9 \%)$ & $3(10.7 \%)$ & $6(21.4 \%)$ & $1(3.6 \%)$ & $1(3.6 \%)$ & $0(0.0 \%)$ & $1(3.6 \%)$ & $28(43.8 \%)$ \\
\hline Tacrolimus combined with Mycophenolate mofetil & $8(44.4 \%)$ & $10(55.6 \%)$ & $0(0.0 \%)$ & $0(0.0 \%)$ & $0(0.0 \%)$ & $0(0.0 \%)$ & $0(0.0 \%)$ & $0(0.0 \%)$ & $18(28.1 \%)$ \\
\hline $\begin{array}{l}\text { Cyclosporine combined with Cortisone and } \\
\text { Mycophenolate mofetil }\end{array}$ & $2(28.6 \%)$ & $1(14.3 \%)$ & $2(28.6 \%)$ & $1(14.3 \%)$ & $0(0.0 \%)$ & $0(0.0 \%)$ & $1(14.3 \%)$ & $0(0.0 \%)$ & $7(10.9 \%)$ \\
\hline Total & $24(37.5 \%)$ & $21(32.8 \%)$ & $5(7.8 \%)$ & $9(14.0 \%)$ & $1(1.6 \%)$ & $1(1.6 \%)$ & $2(3.1 \%)$ & $1(1.6 \%)$ & $64(100 \%)$ \\
\hline
\end{tabular}

E. H/D: Entamoeba histolytica/dispare, E. C: Entamoeba coli, C. P: Cryptosporidium parvum, G. L: Giardia lambelia, E. V: Enterobius vermicularis, H. N: Hymenolepis nana, A. L: Ascaris lumbricoides, S. S: Strongyloides stercoralis

G. lambelia. Our results agree with previous reported results [12]. Comparing to previous reports on the prevalence of intestinal parasites in developing countries, this results were also in agreement [24, 25]. Also in industrial countries such as Albania [26], Poland [27, 28] and Turkey [29]. However, the higher rates in these communities attributed to improper hygiene and agricultural backgrounds.

Cryptosporidium infection is prevalent in communities with overcrowding and low level sanitation [14], and its prevalence reaches up to $36 \%$ in certain developing countries [30]. Given worldwide distribution and can be transmitted by contaminated food and water $[20,31]$, our results are in-discordant with Udgiri et al. [16], reported the incidence of C. parvum infections in India. They pointed 12 out of 60 patients had Cryptosporidium oocysts., also in Saudi Arabia C. parvum were reported to be the most prevalent intestinal parasite [31]. However, In Sudan the exact coccidian infection rates are still not known.

The prevalence of $H$. nana was found to be $(0.3 \%)$, this finding was in accordance with growing study also with previous reports from Albania [26]. Whereas, only one case of $A$. lumbricoides has been diagnosed in this study albeit there are mounting evidence of studies pertaining to Ascariasis not only among immunocompromised hosts [4] but also across the population of different regions [26-28, 32-34].

The data presented in this study highlighted the distribution of $S$. stercoralis among renal transplant recipients; showing only one case has been diagnosed. Notwithstanding, in emerging body of study, S. stercoralis was reported as the most prevalent parasite, and it can cause an overwhelming disease in transplant recipients [9] with severe type of infection in non immunocompromised patients as reported in France [35] and Spain [36, 37].

Although the recipient was taking Cyclosporine A which known to become a cornerstone in prophylactic immunosuppression. Hopefully, the use of cyclosporine has lessened the incidence of strongyloidiasis in renal transplant recipients [38, 39]. None surprisingly, highdose of corticosteroid among infected donors can increase rates and intensity of S. stercoralis transmission [40].

The data presented in the current study found no conclusive data of difference among transplanted subjects compared to non-transplanted healthy population for harboring parasitic infections. The explanation might lie in the fact that these parasitic infections are normally distributed in the community and transmitted through contaminated food and water resources [20]. This findings may agree with several studies stating that intestinal parasitic infections in immune-compromised patients depend mainly on the frequency of intestinal parasitism in the local community, showing no correlation to immune status of the patients $[13,41]$. Also this finding is similar to results attained from HIV patients in comparison with healthy individuals $[13,42,43]$ and in parasitic infections among renal transplanted patients in comparison with non-transplanted controls in Iran [44].

\section{Conclusions}

Examination of a stool sample will benefit all patients on long-lasting immunosuppressive therapy to prevent the disease's distressing and death and improve their quality of life.

\section{Limitations}

- Presence of the parasitic infection among renal transplanted recipients still unknown whether the exposure occurred before or after the transplantation therefore examining transplant patients before and after the transplant and at different time intervals following transplantation would significantly improve future studies.

- A need for accurate estimate for the parasitic infections across the population is required. 


\section{Authors' contributions}

NSM, EES and AME provided conceptual framework for the project, guidance for interpretation of the data, performed data analysis, NSM, EES, AME, HHS, BAA and MAM participated in the performance of the Parasitological work. NSM, EES, EET and BKE performed the statistical analysis and guidance for data interpretation. All authors read and approved the final manuscript.

\section{Author details}

${ }^{1}$ Department of Parasitology and Medical Entomology, Faculty of Medical Laboratory Sciences, Sinnar University, Sinnar, Sudan. ${ }^{2}$ Department of Molecular Biology, National University Research Institute, National University, Khartoum, Sudan. ${ }^{3}$ Department of Parasitology and Medical Entomology, Faculty of Medical Laboratory Sciences, Nile College, Khartoum, Sudan. ${ }^{4}$ Department of Histopathology and Cytology, Faculty of Medical Laboratory Sciences, University of Khartoum, Khartoum, Sudan. ${ }^{5}$ Mycetoma Research Center, University of Khartoum, Khartoum, Sudan. ${ }^{6}$ Department of Histopathology and Cytology, Ibn Sina University, Khartoum, Sudan. ${ }^{7}$ Department of Histopathology and Cytology, Faculty of Medical Laboratory Sciences, Port Sudan AL-Ahlia College, Port Sudan, Sudan. ${ }^{8}$ Department of Biostatistics and Central Supervision Unit, Ministry of Health, Riyadh, Kingdom of Saudi Arabia. ${ }^{9}$ Department of Medical Microbiology, Faculty of Medical Laboratory Science, University of Khartoum, Khartoum, Sudan. ${ }^{10}$ Department of Basic Sciences, College of Medicine, University of Bisha, Bisha, Kingdom of Saudi Arabia.

${ }^{11}$ Department of Histopathology and Cytology, Faculty of Applied Medical Sciences, University of Bisha, Bisha, Kingdom of Saudi Arabia.

\section{Acknowledgements}

We are of great thanks for kind collaboration and assistance of the clinical staff of Sudanese Kidney Association hospital in Khartoum state, Sudan during patients' reception and sampling. And also great thanks to all participants contributed to this work.

\section{Competing interests}

The authors declare that they have no competing interests.

\section{Availability of data and materials}

The datasets used and/or analyzed during the current study are available from the corresponding author on reasonable request.

\section{Consent to publish}

Not applicable.

\section{Ethics approval and consent to participate}

The study was approved by the faculty research board of Medical Laboratory Sciences Review Board of the University of Khartoum, Sudan, for the hospital administration of patients and control groups. Control group was recruited randomly form healthy individuals. Informed consent was obtained using written consent from adults, parents or legal custodians of children.

\section{Funding}

Not applicable.

\section{Publisher's Note}

Springer Nature remains neutral with regard to jurisdictional claims in published maps and institutional affiliations.

Received: 29 March 2018 Accepted: 20 August 2018

Published online: 29 August 2018

\section{References}

1. Schwartz B, Mawhorter S. Parasitic infections in solid organ transplantation. Am J Transplant. 2013;13(s4):280-303.

2. Chapman JR. What are the key challenges we face in kidney transplantation today? Transplant Res. 2013;2(1):S1.

3. Abecassis M, Bartlett ST, Collins AJ, Davis CL, Delmonico FL, Friedewald $\mathrm{J}$, et al. Kidney transplantation as primary therapy for end-stage renal disease: a National Kidney Foundation/Kidney Disease Outcomes
Quality Initiative (NKF/KDOQI ${ }^{\mathrm{TM}}$ ) conference. Clin J Am Soc Nephrol. 2008;3(2):471-80.

4. Bhatti $A B$, Usman M. Chronic renal transplant rejection and possible antiproliferative drug targets. Cureus. 2015;7(11):e376.

5. Thom K, Forrest G. Gastrointestinal infections in immunocompromised hosts. Curr Opin Gastroenterol. 2006;22(1):18-23.

6. Barsoum RS. Parasitic infections in organ transplantation. Exp Clin Transplant. 2004;2(2):258-67.

7. Barsoum RS. Parasitic infections in transplant recipients. Nat Rev Nephrol. 2006;2(9):490.

8. Kotton C, Lattes R. Parasitic infections in solid organ transplant recipients. Am J Transplant. 2009:9(s4):S234-51.

9. Ko KS, Cho DO, Ahn JH, Lee TW, Ihm CG, Chang SG, et al. Infections after renal transplantation. Transplant Proc. 1994:26(4):2072-4.

10. Snyder JJ, Israni AK, Peng Y, Zhang L, Simon TA, Kasiske BL. Rates of first infection following kidney transplant in the United States. Kidney Int. 2009;75(3):317-26.

11. Azami M, Sharifi M, Hejazi SH, Tazhibi M. Intestinal parasitic infections in renal transplant recipients. Braz J Infect Dis. 2010;14(1):15-8.

12. Valar C, Keitel E, Dal Pra R, Gnatta D, Santos A, Bianco P, et al. Parasitic infection in renal transplant recipients. Transplant Proc. 2007;39:460-2.

13. Meamar A, Rezaian M, Mohraz M, Zahabian F, Hadighi R, Kia E. A comparative analysis of intestinal parasitic infections between HIV+/AIDS patients and non-HIV infected individuals. Iran J Parasitol. 2007;2(1):1-6.

14. Brink A-K, Mahe C, Watera C, Lugada E, Gilks C, Whitworth J, et al. Diarrhoea, CD4 counts and enteric infections in a community-based cohort of HIV-infected adults in Uganda. J Infect. 2002;45(2):99-106.

15. Keiser PB, Nutman TB. Strongyloides stercoralis in the immunocompromised population. Clin Microbiol Rev. 2004;17(1):208-17.

16. Udgiri N, Minz M, Kashyap R, Heer M, Gupta C, Mohandas K, et al. Intestinal cryptosporidiasis in living related renal transplant recipients. Transplant Proc. 2004;36:2128-9.

17. Ali M, Mahmoud L, Abaza B, Ramadan M. Intestinal spore-forming protozoa among patients suffering from chronic renal failure. J Egypt Soc Parasitol. 2000:30(1):93-100.

18. Chieffi PP, Sens YA, Paschoalotti MA, Miorin LA, Silva HGC, Jabur P. Infection by Cryptosporidium parvum in renal patients submitted to renal transplant or hemodialysis. Rev Soc Bras Med Trop. 1998;31(4):333-7.

19. Minz M, Udgiri NK, Heer MK, Kashyap R, Malla N. Cryptosporidiasis in live related renal transplant recipients: a single center experience. Transplantation. 2004;77(12):1916-7.

20. Mohamed MA, Siddig EE, Elaagip AH, Edris AMM, Nasr AA. Parasitic contamination of fresh vegetables sold at central markets in Khartoum state, Sudan. Ann Clin Microbiol Antimicrob. 2016:15(1):17.

21. Bashier L. Frequency of intestinal parasites with emphasis on opportunistic parasites among renal transplant recipients in Sudan: UOFK; 2015.

22. Organization $\mathbf{W H}$. Bench aids for the diagnosis of intestinal parasites. Geneva: World Health Organization; 1994.

23. Casemore D. Laboratory methods for diagnosing cryptosporidiosis. J Clin Pathol. 1995;48:128.

24. Kang G, Mathew MS, Prasanna Rajan D, Daniel JD, Mathan MM, Mathan V, et al. Prevalence of intestinal parasites in rural Southern Indians. Trop Med Int Health. 1998:3(1):70-5.

25. Gbakima A, Sahr F. Intestinal parasitic infections among rural farming communities in eastern Sierra Leone. Afr J Med Med Sci. 1995;24(2):195-200.

26. Spinelli R, Brandonisio O, Serio G, Trerotoli P, Ghezzani F, Carito V, et al. Intestinal parasites in healthy subjects in Albania. Eur J Epidemiol. 2006;21(2):161-6.

27. Biaduń W, Chybowski J, Rukasz H, Stanios H. Occurence of gastrointestinal parasites in children in Lublin region in the period 1976-2000. Wiad Parazytol. 2001;47(3):417-22.

28. Bitkowska E, Wnukowska N, Wojtyniak B, Dzbeński T. Occurrence of intestinal parasites among first grade students in Poland in years 2002/2003. Przegl Epidemiol. 2004;58(2):295-302.

29. Aksoy Ü, Akisu C, Delibas SB, Ozkoç S, Sahin S, Usluca S. Demographic status and prevalence of intestinal parasitic infections in schoolchildren in Izmir, Turkey. Turk J Pediatr. 2007:49(3):278. 
30. Gomez MM, Atzori C, Ludovisi A, Rossi P, Scaglia M, Pozio E. Opportunistic and non-opportunistic parasites in HIV-positive and negative patients with diarrhoea in Tanzania. Trop Med Parasitol. 1995;46(2):109-14.

31. Amer $\mathrm{OH}$, Ashankyty IM, Haouas NAS. Prevalence of intestinal parasite infections among patients in local public hospitals of Hail, Northwestern Saudi Arabia. Asian Pac J Trop Med. 2016;9(1):44-8.

32. Avetisian L. Epidemiological surveillance of parasitic diseases in the republic of Armenia. Med Parazitol. 2004;1:21-4.

33. Małafiej $E$, Spiewak E. Serological investigation in children infected with Ascaris lumbricoides. Wiad Parazytol. 2001;47(4):585-90.

34. Ulukanligil M, Seyrek A, Aslan G, Ozbilge H, Atay S. Environmental pollution with soil-transmitted helminths in Sanliurfa, Turkey. Mem Inst Oswaldo Cruz. 2001;96(7):903-9.

35. Magnaval J-F, Mansuy J-M, Villeneuve L, Cassaing S. A retrospective study of autochthonous strongyloïdiasis in Région Midi-Pyrénées (Southwestern France). Eur J Epidemiol. 2000;16(2):179-82.

36. Sanchez PR, Guzman AP, Guillen SM, Adell RI, Estruch AM, Gonzalo IN, et al. Endemic strongyloidiasis on the Spanish Mediterranean coast. QJM. 2001;94(7):357-63.

37. Roman-Sanchez P, Pastor-Guzman A, Moreno-Guillen S, Igual-Adell R, Er-Generoso SS, Tornero-Estebanez C. High prevalence of Strongyloides stercoralis among farm workers on the Mediterranean coast of Spain: analysis of the predictive factors of infection in developed countries. Am J Trop Med Hyg. 2003;69(3):336-40.

38. Snydman DR, Walker M, Zunt JR. Parasitic central nervous system infections in immunocompromised hosts. Clin Infect Dis. 2005;40(7):1005-15.

39. Fiorelli A, Stolf N, Honorato R, Bocchi E, Bacal F, Uip D, et al. Later evolution after cardiac transplantation in Chagas' disease. Transplant Proc. 2005;37:2793-8.

40. Hamilton KW, Abt PL, Rosenbach MA, Bleicher MB, Levine MS, Mehta J, et al. Donor-derived Strongyloides stercoralis infections in renal transplant recipients. Transplantation. 2011;91(9):1019-24.

41. Cotte L, Rabodonirina M, Piens M, Perreard M, Mojon M, Trepo C. Prevalence of intestinal protozoans in French patients infected with HIV. JAIDS J Acquir Immune Defic Syndr. 1993;6(9):1024-9.

42. Zali MR, Mehr AJ, Rezaian M, Meamar AR, Vaziri S, Mohraz M. Prevalence of intestinal parasitic pathogens among HIV-positive individuals in Iran. Jpn J Infect Dis. 2004;57(6):268-70.

43. Hailemariam G, Kassu A, Abebe G, Abate E, Damte D, Mekonnen E, et al. Intestinal parasitic infections in HIV/AIDS and HIV seronegative individuals in a teaching hospital, Ethiopia. Jpn J Infect Dis. 2004;57(2):41-3.

44. Rostami MN, Keshavarz H, Eskandari E, Kia E, Rezaeian M. Intestinal parasitic infections in renal transplant recipients. Iran J Parasitol. 2007;2(3):16-23.
Ready to submit your research? Choose BMC and benefit from:

- fast, convenient online submission

- thorough peer review by experienced researchers in your field

- rapid publication on acceptance

- support for research data, including large and complex data types

- gold Open Access which fosters wider collaboration and increased citations

- maximum visibility for your research: over 100M website views per year

At BMC, research is always in progress.

Learn more biomedcentral.com/submissions 